\title{
Hardware Design of Dynamic Reactive Power Compensation Control System
}

\author{
Yi Tu ${ }^{a}$, Jing Chen ${ }^{b}$, Youxin Yuan ${ }^{c}$, Xuesong Zhou ${ }^{d}$ \\ School of Automation, Wuhan University of Technology, Wuhan, 430070, China \\ aemail: 752294211@qq.com, ${ }^{\mathrm{b}}$ email: \\ jingchen680@163.com, 'email:YYX2000@263.net,_ demail: 86382703@qq.com
}

Keywords: reactive power compensation; dynamic; hardware design

\begin{abstract}
The composite power loads largely need the reactive power. It leads to the decrease of power factor in power grid. The traditional static reactive power compensator is difficult to provide the reactive power compensation for the composite load reactive. Therefore, a dynamic reactive power compensation method of composite power load has been proposed by the author. Based on this method, the hardware of the dynamic reactive power compensation control system is designed in this paper. The following works have been done in the study: the design requirements, the control system components, and the hardware design of dynamic reactive power compensation control system. The hardware circuit designed in this paper has been applied extensively in the dynamic reactive power compensation device.
\end{abstract}

\section{Introduction}

In recent years, the reactive power is largely consumed by the composite power loads. It leads to the decrease of the power factor and voltage in the power grid, and even influences the whole gird. Therefore, method of applying static electric capacity to compensate the reactive power is used by most consumers. Apparently, with the huge change of industrial environment, the traditional static reactive power compensator is difficult to provide the reactive power compensation for the composite load reactive [1][2].

Therefore, a dynamic reactive power compensation method of the composite power load has been proposed by the author [3]-[5]. According to the method and design requirements of the dynamic reactive power compensation control system, the hardware of the dynamic reactive power compensation control system is designed in this paper. The hardware design mainly includes PLC input and output circuits, and electric parameter acquisition circuit. The hardware circuit designed in this paper has been applied extensively in the dynamic reactive power compensation device.

\section{Design Requirements of Dynamic Reactive Power Compensation Control System}

According to the topological structure of dynamic reactive power compensation device, the design requirements of dynamic reactive power compensation control system are presented.

The topology of the dynamic reactive power compensation device is shown in Fig. 1. QS is the high-voltage isolation switch; QF is the high-voltage circuit breaker; KM1, KM2 are high-voltage connectors; the capacitor bank consists of capacitor C1-n, high voltage connectors KM11-1n, and high voltage fuse FU1-n. N values range is set according to the needs. 


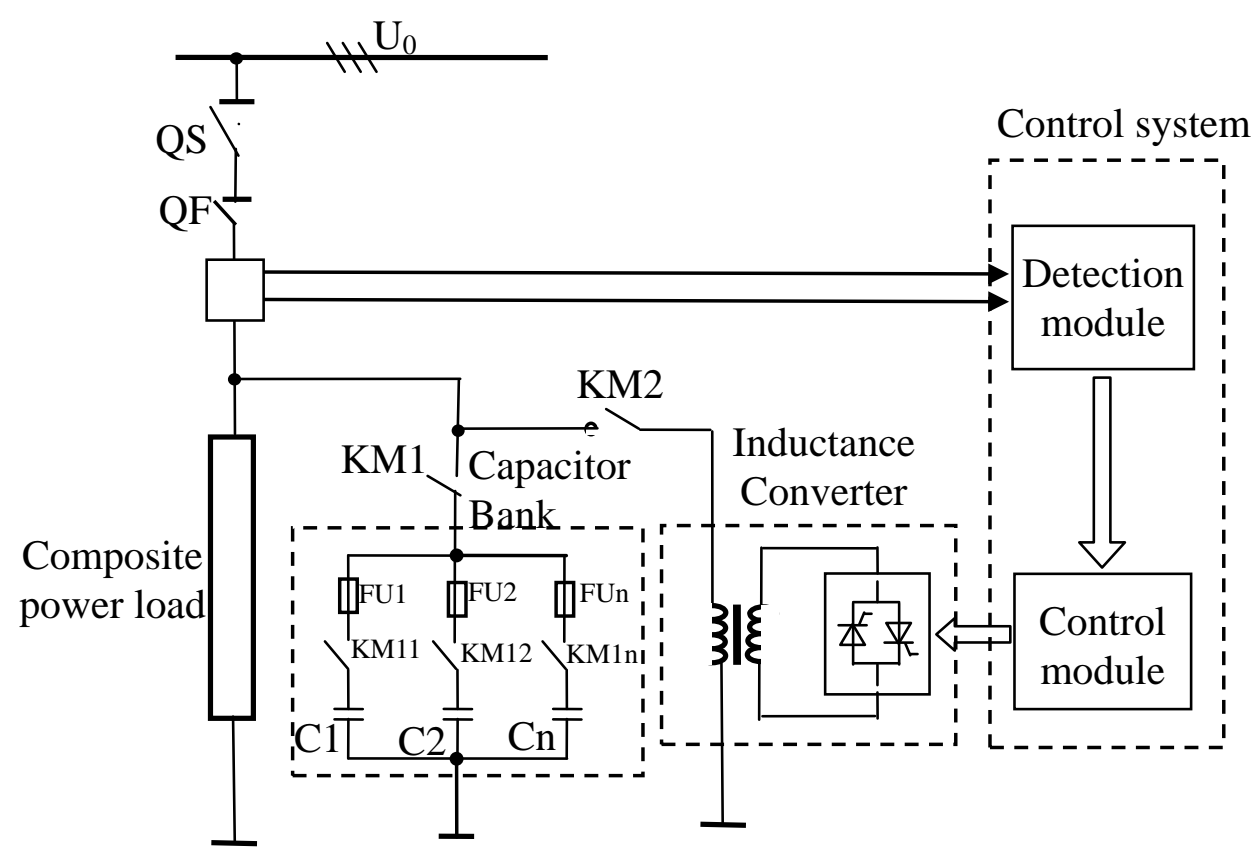

Fig.1. The topology of the dynamic reactive power compensation

The design requirements of dynamic reactive power compensation control system are as follows:

Power factors. According to the composite load, the power factors are adjusted to the range of 0.92 0.98 by switching on/off appropriate capacitor banks. When the load needs different reactive power, the needed reactive power compensation can be calculated according to real-time data.

Fault monitoring. The faults of the control system are monitored and the warnings are given. When the system is over-current and over-voltage, sound and light alarm signal can be given. Meanwhile, corresponding measures is also taken to protect the system.

\section{Control System Components of Dynamic Reactive Power Compensation}

According to the design requirements of dynamic reactive power compensation control system, PLC is chosen as the controller. The main circuit is the important part of the dynamic reactive power compensation control system. This control system consists of HMI, current and voltage transformer, electrical parameters acquisition, PLC controller, I/O interface, D/A conversion. The block diagram of the control system components is shown in Fig. 2.

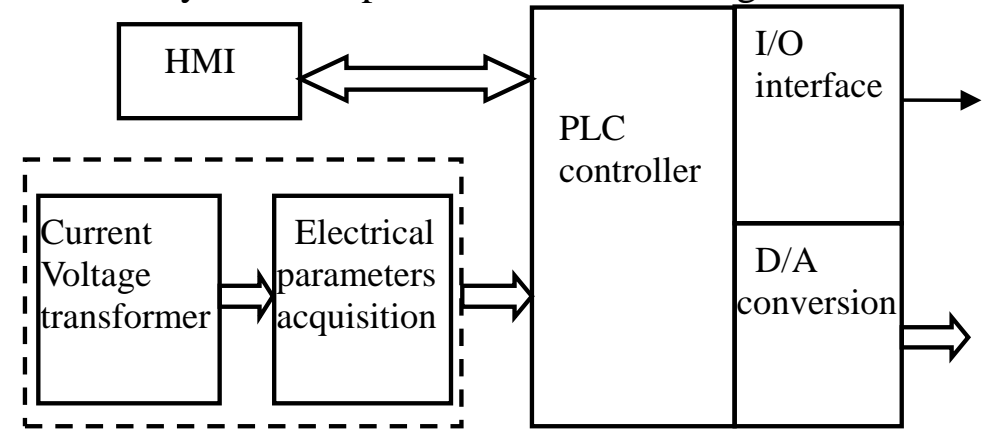

Fig.2. The block diagram of the control system components

In the Fig.2, the model of PLC controller is S7-200 CPU22. Its integrated input points is 24, the input signal voltage is DC $24 \mathrm{~V}$ and the input current is $4 \mathrm{~mA}$. In the D/A conversion, EM235 module is chosen as the extended analog output module. The WB1867B35-1 electronic energy acquisition module is chosen as the electrical parameters acquisition module. 


\section{Hardware Design of Dynamic Reactive Power Compensation Control System}

The hardware design of dynamic reactive power compensation control system mainly includes the digital input circuit, the digital output circuit, and the electrical parameters acquisition circuit.

Digital input circuit. In the Fig.3, the input of reactive power compensation control system is switch signal. And it's mainly generated by buttons of SB1 and SB2, high-voltage connectors of KM2, KM11, KM12 and KM13, high-voltage circuit breaker QF. The QF is the power switch of the main circuit. KM11, KM12 and KM13 are used to control the switching of the capacitor banks. KM2 is used to control the inductance converter. Therefore, the on-off state and control instructions are inputted to PLC. The digital input interface wiring diagram of PLC is shown in Fig.3.

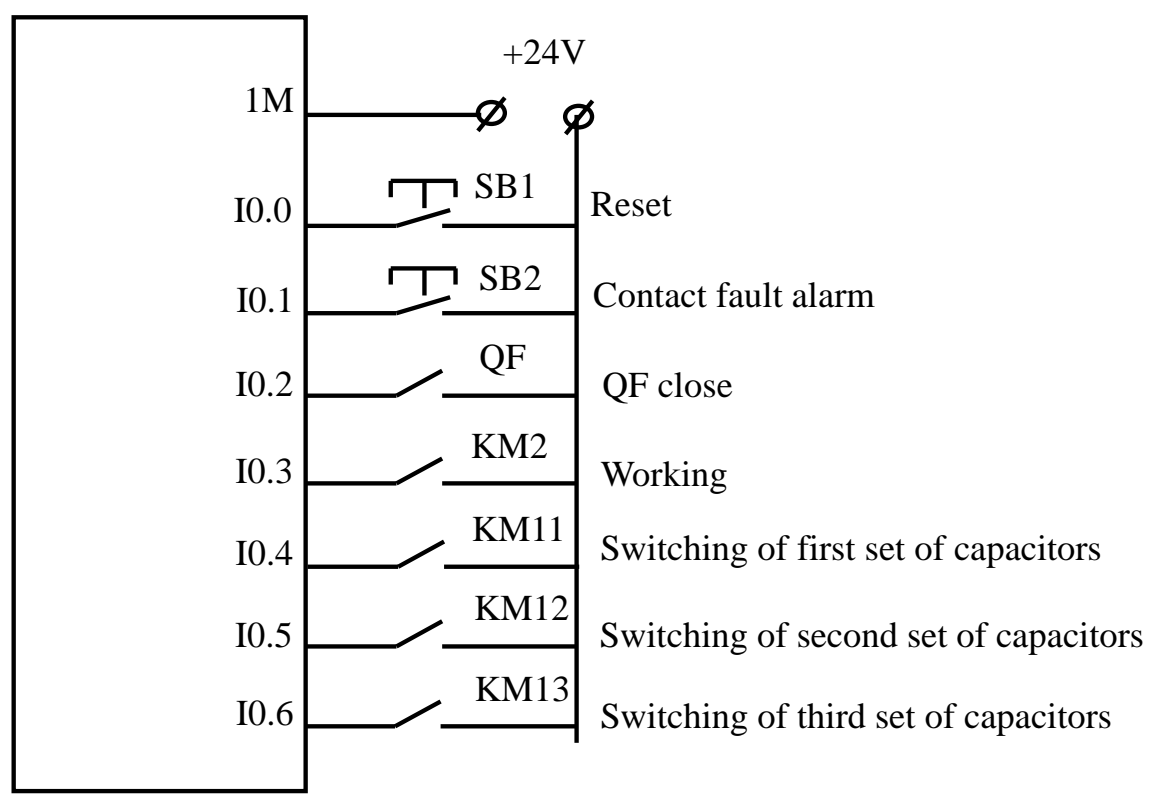

Fig.3. The digital input interface wiring diagram

Digital output circuit. As shown in the Fig.4, six output ports of PLC are used in the digital output circuit. The 6J1-6J6 are AC intermediate relays. The loads driven by the output of PLC are mostly the connectors. Owing to the connector is high-current, it's driven by the intermediate relay. The capacitor banks are switched on/off by the high-voltage connectors of KM11, KM12 and $\mathrm{KM} 13$, and the KM11, KM12 and KM13 are driven by the AC intermediate relays of 6J1, $6 \mathrm{~J} 2$ and $6 \mathrm{~J} 3$ respectively. The 6J1, 6J2 and 6J3 are driven by the ports of Q0.0, Q0.1 and Q0.2 respectively. The inductance converter is controlled by the high-voltage connector KM2, and the KM2 is driven by the AC intermediate relays of 6J4. The 6J4 is driven by the Q0.3 port. The Q0.5 port is in reserve, and it is used when the control system is needed to extend other functions. The digital output interface wiring diagram of PLC is shown in Fig.4. 


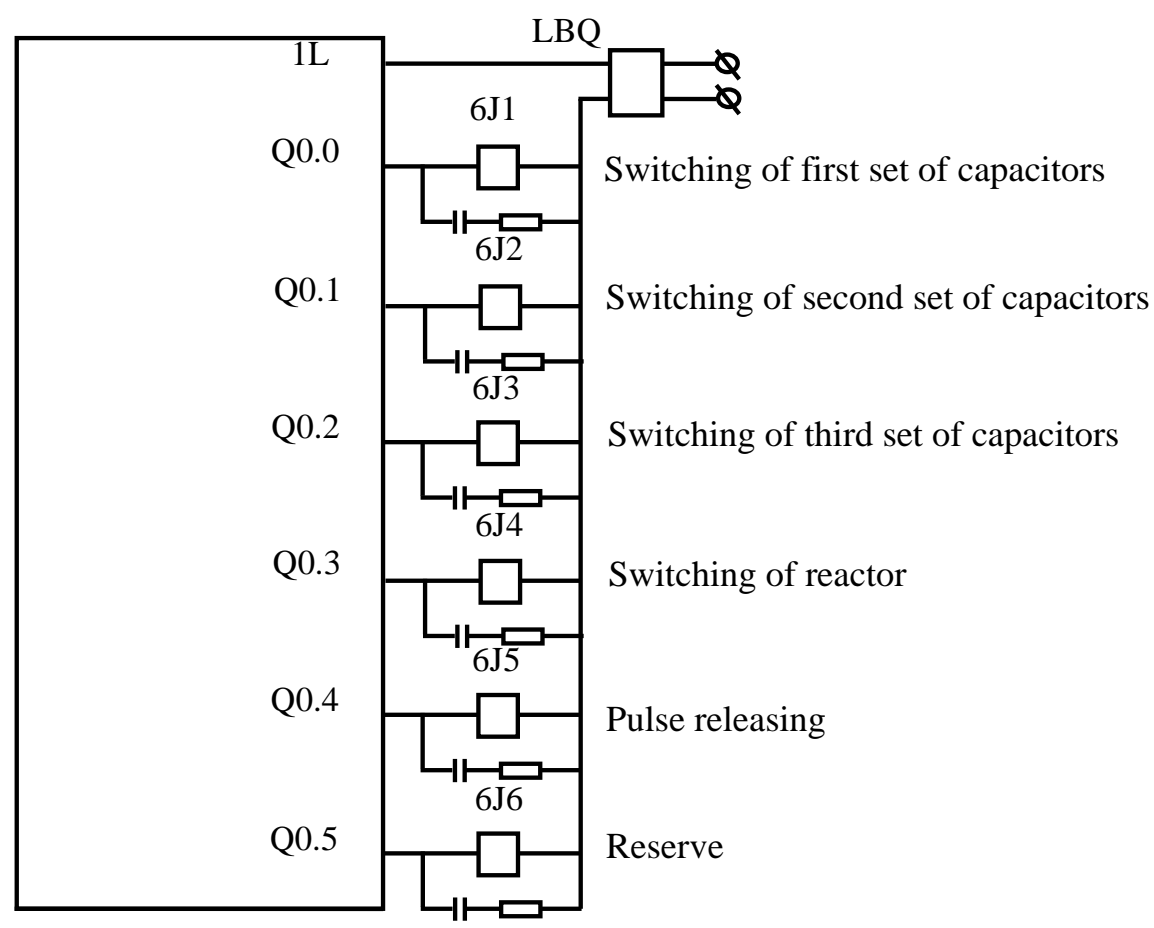

Fig.4. The digital output interface wiring diagram of PLC

Electrical parameters acquisition circuit. The electrical parameters mainly include grid voltage, power factor, active power and reactive power, etc. The electrical parameters acquisition module can complete the acquisition and processing of the data, and can convert the data into digital quantity. Furthermore, the electrical parameters acquisition module can complete the data transmission with CPU real-time communication. The digital signal is sent to PLC by RS-485 bus, and the Modbus-RTU protocol is used to communicate.

\section{Conclusion}

According to the topological structure, the PLC is chosen as controller of control system. And the following works have been done in the study: the design requirements, the control system components, and the hardware design of dynamic reactive power compensation control system. This control system has the advantages of real-time monitoring and dynamic adjustment of reactive power compensation. At present, the hardware circuit designed in this paper has been applied extensively in the dynamic reactive power compensation device.

\section{Acknowledgement}

This work was financially supported by the projects of Hubei Science and Technology Plans of China (Granted No: 2013BAA009).

\section{References}

[1] Jingjing Gao, Yulin Zhao. Overview of the Present Status Development Tendency of Var Compensation Technology of Power System [J]. Journal of Northeast Agricultural University, 2004 35(5) 639-644.

[2] Xiaozhi Yang. Analysis and Comparison of Several Reactive Power Compensation Techniques [J]. Anhui Electric Power, 2006 23(2) 40-42.

[3] Qijian Cheng, Jing Chen, Youxin Yuan, Xuesong Zhou, Shijie Deng. Research on a Dynamic Reactive Power Compensation Method of Composite Power Load [J]. Applied Mechanics and 
Materials, 2014 602-605 2840-2843.

[4] Yifei Wang, Youxin Yuan, Jing Chen, Qijian Cheng. A Dynamic Reactive Power Compensation Method of Super High-Power and High-voltage Motor [J]. Applied Mechanics and Materials, 2014 602-605 2828-2831.

[5] Yongzhong Ding, Youxin Yuan. Research on Variable Reactor Based on Power Convert [J]. Journal of Wuhan University of Technology, 2013 30(3) 75-83. 